This manuscript is a preprint and was submitted for publication to Geophysics. The manuscript has undergone peer-review, but was not accepted for publication. As we are addressing the reviewer's comments, the manuscript may change slightly in the future. When published, the final version of the manuscript will be available via the 'Peer-reviewed Publication DOI' link on the right-hand side of this web page. In the meantime, we are happy to receive feedback. 


\title{
PRINCIPAL COMPONENT ANALYSIS OF SEISMIC FACIES
}

\author{
T. Wrona ${ }^{1,2 *}$, Indranil Pan ${ }^{3,4}$, R.E. Bell ${ }^{5}$, H. Fossen ${ }^{6}$ \& R.L. Gawthorpe \\ 'Department of Earth Science, University of Bergen, Allégaten 41, N-5007 Bergen, Norway. \\ ${ }^{2}$ GFZ German Research Centre for Geosciences, Telegrafenberg, 14473 Potsdam, Germany. \\ ${ }^{3}$ Centre for Process Systems Engineering \& Centre for Environmental Policy, Imperial College London, UK. \\ ${ }^{4}$ The Alan Turing Institute, British Library, London, UK. \\ ${ }^{5}$ Department of Earth Science and Engineering, Imperial College, Prince Consort Road, London, SW7 2BP, UK. \\ ${ }^{6}$ Museum of Natural History, University of Bergen, Allégaten 41, N-5007 Bergen, Norway. \\ *wrona@gfz-potsdam.de
}

\section{ABSTRACT}

Seismic facies analyses are fundamental to the study of sedimentary, tectonic and magmatic systems using seismic reflection data. These analyses generally assume that seismic facies are: (1) well defined, (2) distinct and (3) prevalent patterns in the data. Here, we examine these assumptions critically. First, we demonstrate how to extract the main seismic facies from conventional industry seismic reflection data using principal component analysis. Applying principal component analysis on a large number (up to 1000000$)$ of windows (150×150 samples) reveals typical seismic facies showing: (1) horizontal, (2) dipping, (3) displaced and (4) crisscrossing reflections. These seismic facies are distinct in the sense that the principal components are orthogonal to one another, i.e. we cannot express any one component as a linear combination of the others. Next, we show that a small number of seismic facies (100) can explain most of the variance in the data (>0.6); an assumption that is critical to seismic facies analyses. Lastly, we show a simple way to map these facies across a seismic section. 


\section{INTRODUCTION}

Seismic reflection data provides a key source of information in numerous fields of geoscience, including sedimentology and stratigraphy (e.g., Vail, 1987; Posamentier, 2004), structural geology (Morley, 2002; Baudon and Cartwright, 2008), geomorphology (e.g., Posamentier and Kolla, 2003; Cartwright and Huuse, 2005; Bull et al., 2009) and volcanology (e.g., Hansen et al., 2004; Planke et al., 2005). Seismic facies analyses are an important component of seismic interpretations, in particular when individual reflections become difficult to trace. In these cases, seismic facies patterns are traditionally qualitatively assessed and mapped by expert seismic interpreters (e.g. Payton, 1977; Sheriff, 1980; Bally, 1987; Vail, 1987; Van Wagoner et al., 1987). While the human ability to recognize patterns is extraordinary, it does require significant amounts of time, experience, and expertise from interpreters (e.g., Bond et al., 2012; Bond, 2015; Macrae et al., 2016). Quantitative interpretation techniques involving seismic attributes and machine learning have significantly improved in terms of quality and speed (e.g. Coléou et al., 2003; Chopra and Marfurt, 2008). In line with this development, we investigate if it is possible to derive key seismic facies quantitatively from seismic reflection data.

A seismic facies is defined as the character of a group of reflections involving amplitude, abundance, continuity, and configuration of reflections (Sheriff, 2002). Reflection amplitude, abundance and continuity are typically described qualitatively as high, medium and low or quantified using seismic attributes (e.g. Marfurt et al., 1998; Chopra and Marfurt, 2007). Reflection configurations typically include: (1) parallel, (2) subparallel, (3) divergent, (4) sigmoidal, (5) oblique and (6) hummocky reflections (Sheriff, 1980) and are usually difficult to capture numerically. Most 
conventional seismic interpretations involve a description of the seismic facies observed in the dataset followed by mapping of these seismic facies throughout the dataset (e.g. Roksandić, 1978). This approach requires the seismic facies to be: (1) well defined, (2) distinct from one another and (3) prevalent in the data. This study examines if these assumptions are reasonable using a quantitative workflow based on principal component analysis.
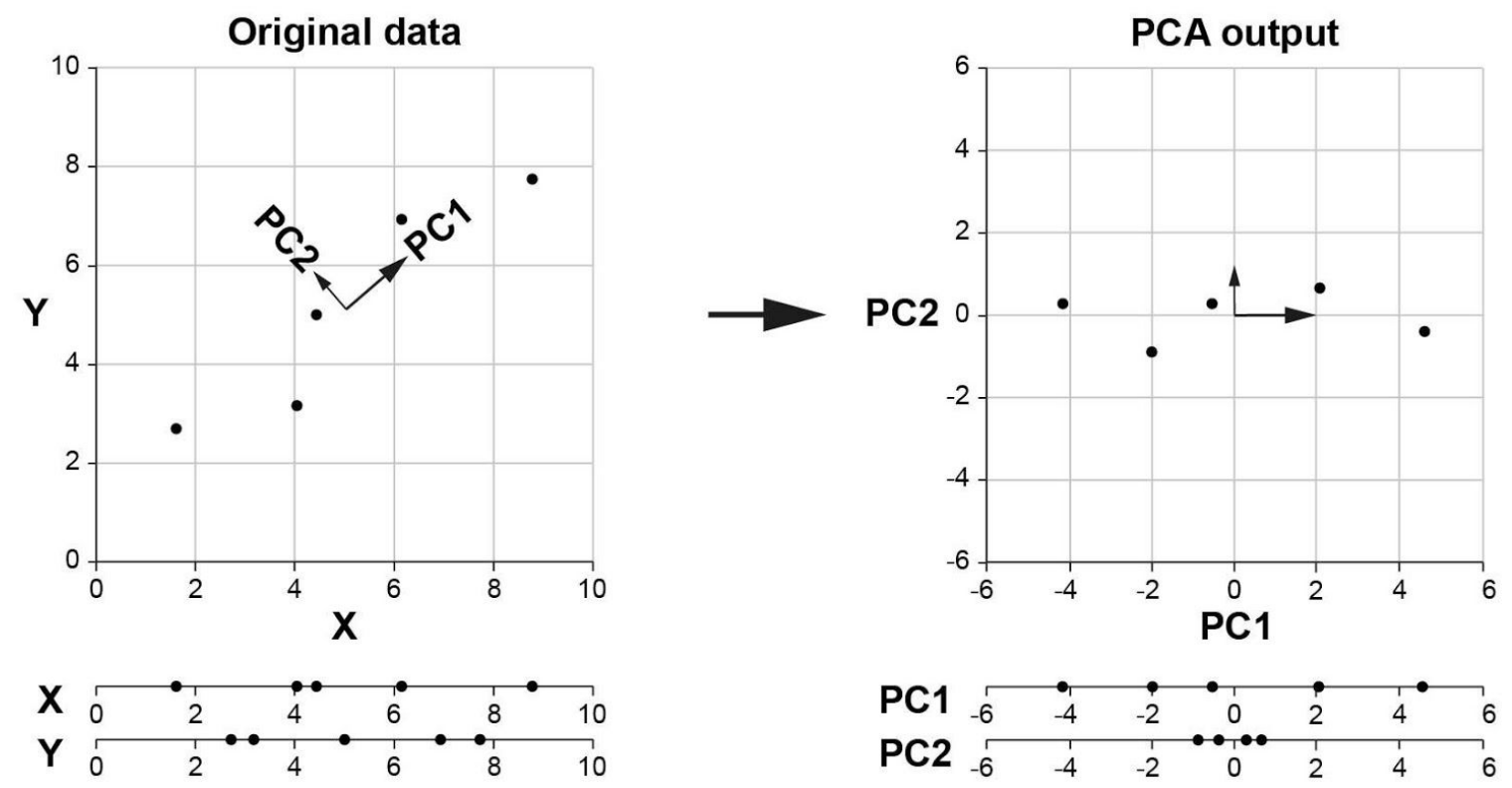

Figure 1: Simple 2-D example illustrating how the principal components reveal the direction of maximum variance in the data. This example is based on visualization by setosa.io/ev/principal-component-analysis/.

Principal component analysis (PCA) is one of the standard procedures of exploratory data analysis (e.g. Jolliffe, 1986). We can think of principal components as alternative coordinate axes, which highlight strong patterns in our data. A simple 2-D example illustrates how principal components reveal the direction of maximum variance (Figure 1), where variance describes how spread out the data is. Applying PCA to up to a million windows randomly extracted from seismic reflection data 
reveals typical seismic facies showing: (1) horizontal, (2) dipping, (3) displaced and (4) crisscrossing reflections. These seismic facies are distinct in the sense that the principal components are orthogonal, i.e. we cannot express any one component as a linear combination of the others. The seismic facies are also prevalent in the data, as they explain most of the variance (>0.6). Finally, we can even produce a simple facies map by projecting the data on to the principal components. As such, this study highlights that the basic assumptions of seismic facies analyses, i.e. that seismic facies are: (1) well defined, (2) distinct and (3) prevalent in the data are valid; an important requirement for conventional and automated seismic facies analyses.

\section{3-D SEISMIC REFLECTION DATA}
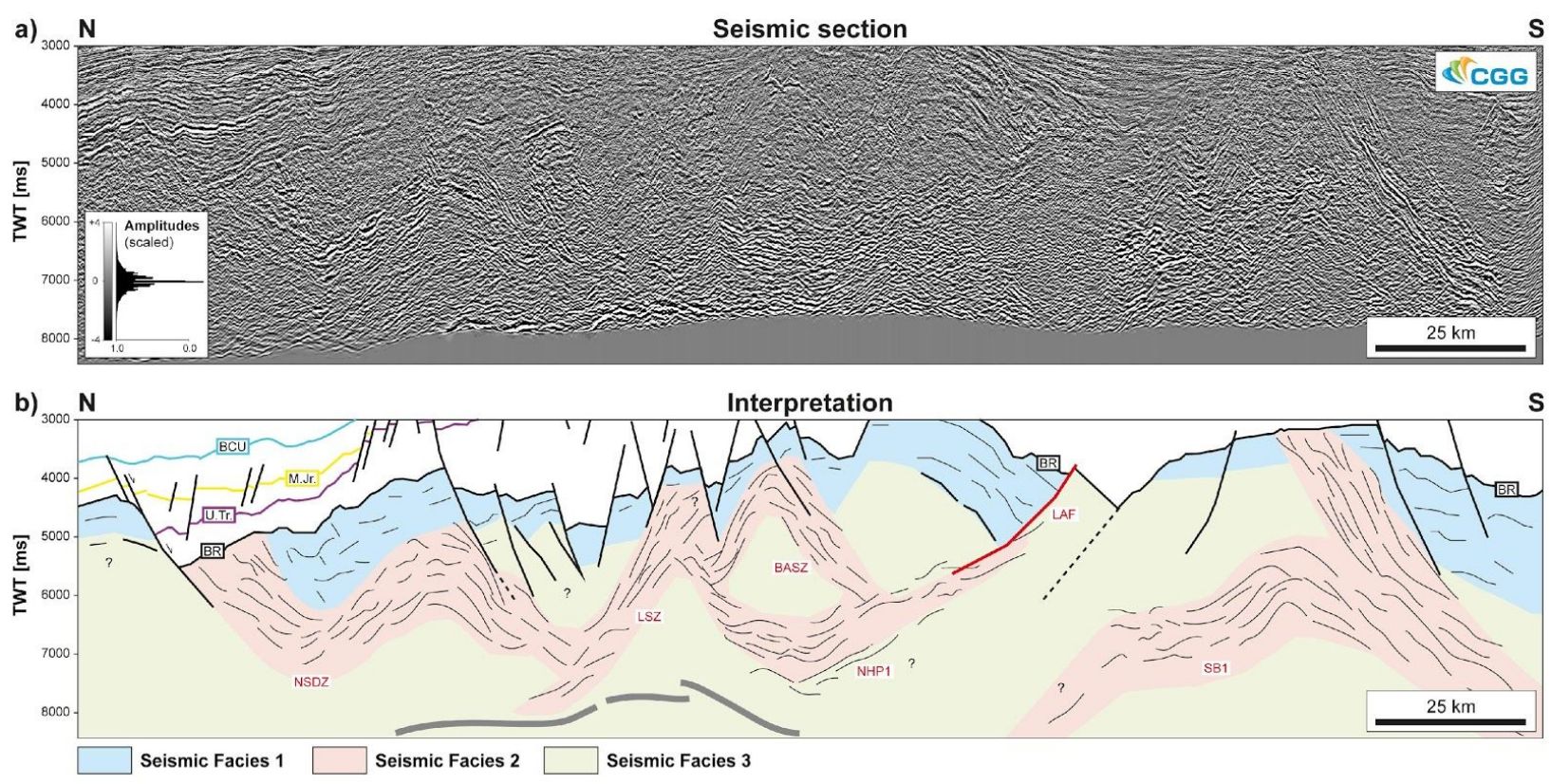

Figure 2: a) 2-D seismic section (courtesy of CGG) with b) geological interpretation by Fazlikhani et al., (2017) with BCU: Base Cretaceous Unconformity; M.Jr.: Middle Jurassic; U.Tr.: Upper Triassic; Base rift surface: NSDZ: Nordfjord-Sogn Detachment Zone; LSZ: Lomre Shear Zone; BASZ: Bergen Arc Shear Zone; NHPI: Northern Horda Platform 1; LAF: Low angle fault; SB1: Stord Basin 1. 
This study uses state-of-the-art 3-D broadband seismic reflection data (courtesy of CGG) from the northern North Sea (Figure 2). The data covers an area of $35,410 \mathrm{~km}^{2}$ and was acquired using a series of up to 8-km-long streamers towed $\sim 40$ $m$ deep. The data recording extends down to $9 \mathrm{~s}$ with a time sampling of $4 \mathrm{~ms}$. The data covers a broad range of frequencies reaching from 2.5 to $155 \mathrm{~Hz}$ (Firth et al., 2014). The binning size was $12.5 \times 18.75 \mathrm{~m}$. The seismic volume was zero-phase processed with SEG normal polarity, i.e. a positive reflection (white) corresponds to an acoustic impedance increase with depth. The data was pre-stack depth-migrated and subsequently stretched to the time domain.

\section{PRINCIPAL COMPONENT ANALYSIS (PCA)}

Principal component analysis (PCA) is a technique used to emphasize variation and highlight patterns in a dataset (e.g. Wold et al., 1987; Turk and Pentland, 1991). For this purpose, PCA converts the original dataset $(X)$ into a new dataset $(Y)$ using a linear transformation $(P)$ :

$$
P X=Y
$$

The goal of the linear transformation $(P)$ is to remove redundancy from the data (Shlens 2014). This is accomplished by diagonalizing the covariance matrix of the new dataset $\left(S_{Y}\right)$ :

$$
S_{Y}=\frac{1}{n-1} Y Y^{T}
$$

We can rewrite $S_{Y}$ using $P$ :

$$
S_{Y}=\frac{1}{n-1} P A P^{T}
$$

where $A=X X^{T}$ and is thus symmetric. A symmetric matrix $A$ is:

$$
A=E D E^{T}
$$


where $D$ is a diagonal matrix and is the matrix of eigenvectors of $A$. Selecting $P \equiv E^{T}$ and substituting Equation 4 into 3 provides:

$$
\begin{gathered}
S_{Y}=\frac{1}{n-1} P\left(P^{T} D P\right) P^{T} \\
S_{Y}=\frac{1}{n-1} D
\end{gathered}
$$

This selection of $P$ diagonalizes the covariance matrix $\left(S_{Y}\right)$. The principal components of the data appear as the eigenvectors of $A=X X^{T}$ and the rows of $P$. Moreover, the variance of $X$ along the principal components are the eigenvalues of $S_{Y}$. The analysis is implemented in Python using the scikit-learn package (Pedregosa et al., 2011) (see Appendix).

In this study, we apply PCA to a 2-D seismic section showing different seismic facies in the basement (Figure 2). Applying PCA to the entire 3-D seismic volume (1.3 TB) is impractical and, as we will see, not necessary to extract the main seismic facies from the data. Instead, we analyze a large number of windows randomly selected from a 2-D seismic section (Figure 2). During PCA, we can set: (1) the scale of the data, (2) the number of principal components, (3) the window size and (4) the number of windows. To explore the effects of these parameters, we conduct a sensitivity analysis. First, we perform PCA using standardized $(\mu=0, \sigma=1)$ or unstandardized data ( $\mu=12, \sigma=18429$ 696) (Figure 3). Second, we extract different numbers of principal components (up to 400) from the data (Figures 4, 5). Third, we analyze the effect of windows sizes ranging from $50 \times 50$ to $200 \times 200$ samples (Figures 6, 7). A sample has a size of $12.5 \mathrm{~m}$ (inline), $18.75 \mathrm{~m}$ (crossline) and $4 \mathrm{~ms}$ in two-way traveltime. Finally, we explore how varying the number of windows (1000 to 1000 000) extracted from the 2-D section affects our results (Figures 8, 9). 


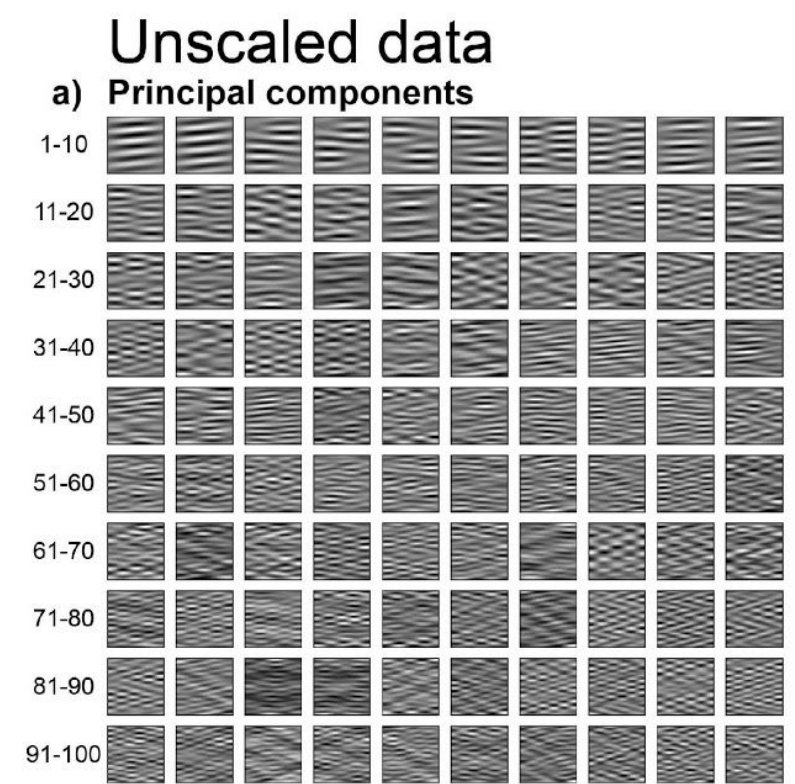

c)

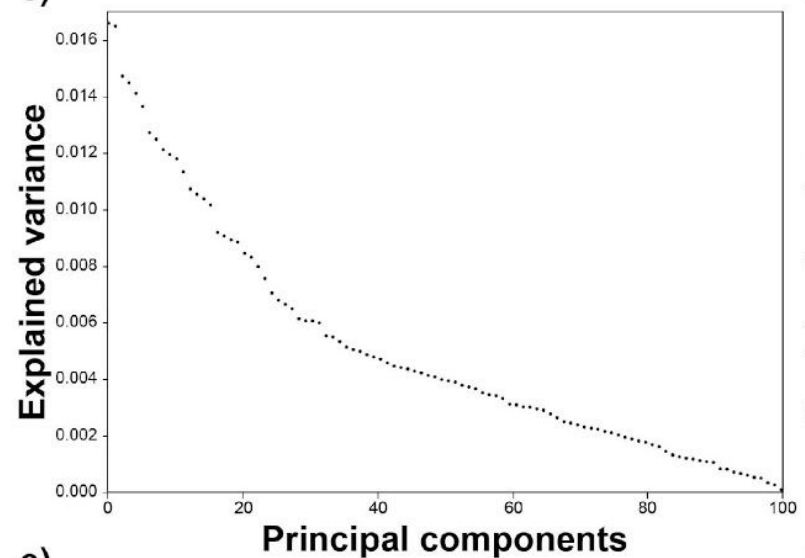

e)

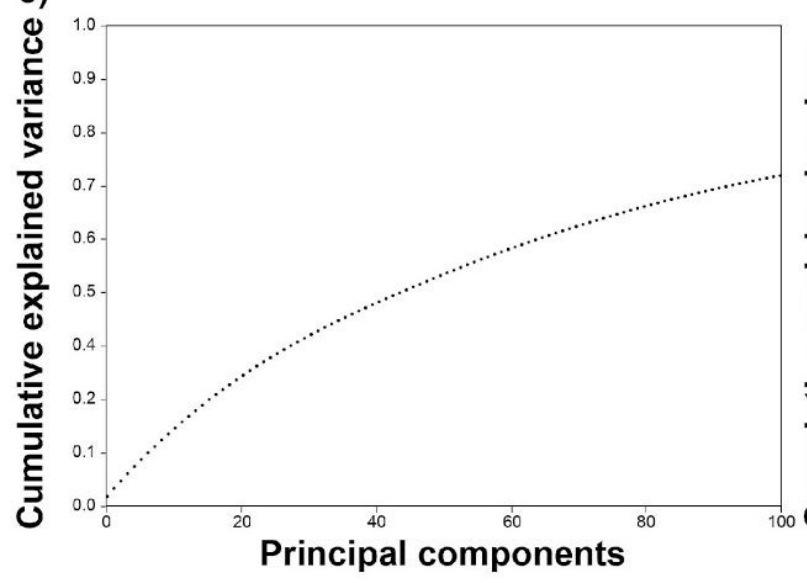

Scaled data

b) Principal components

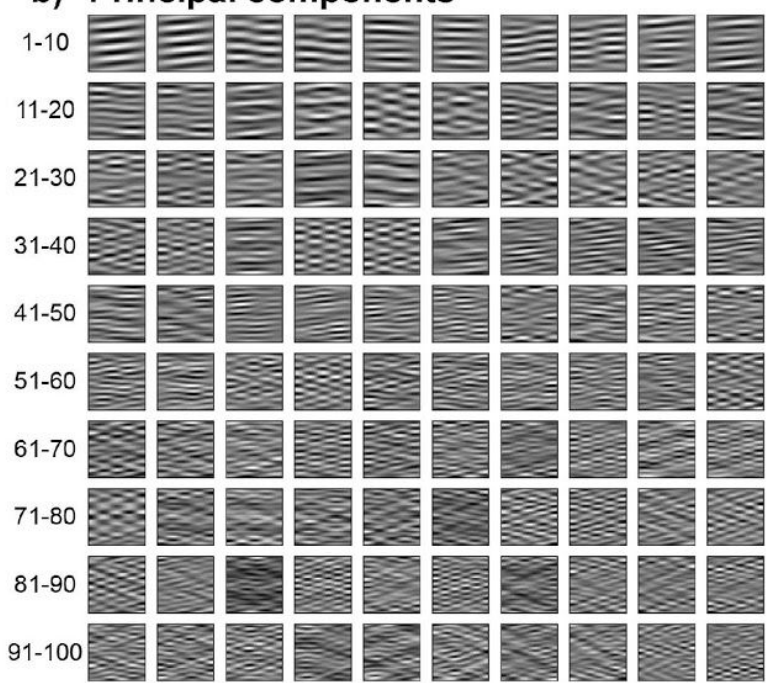

d)

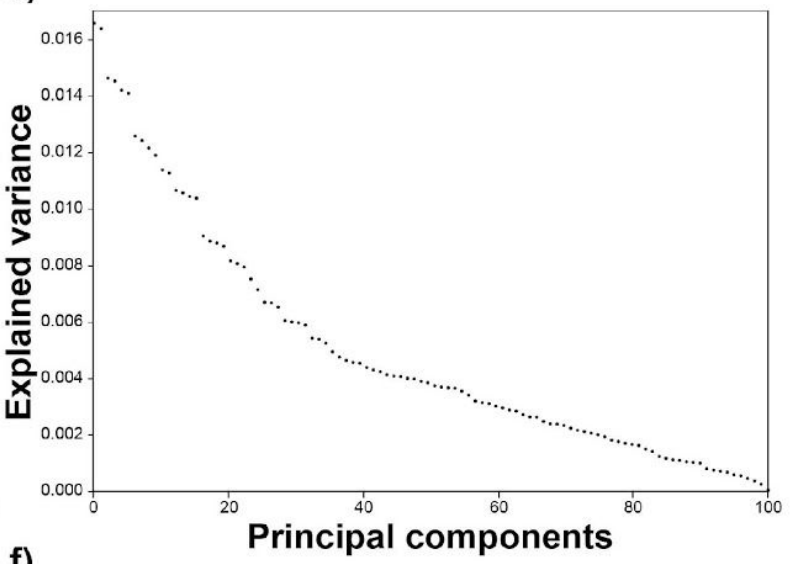

f)

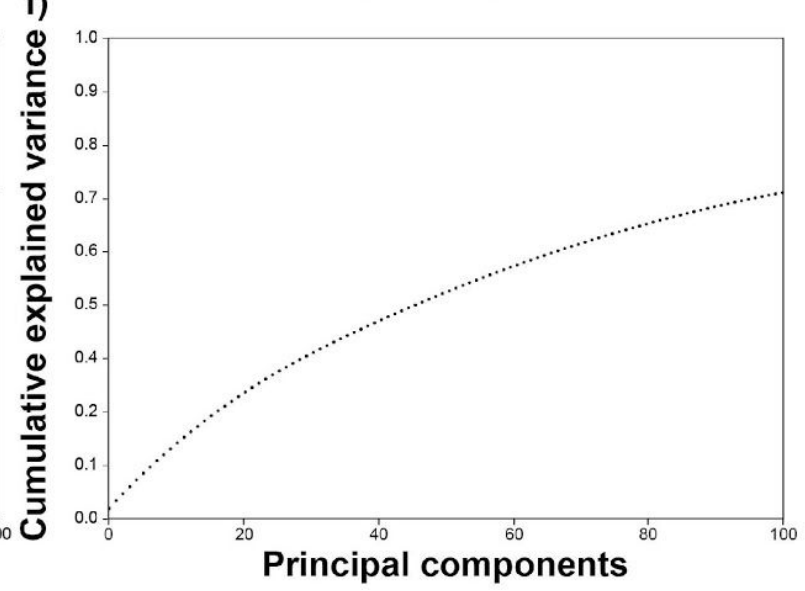

Figure 3: Principal components extracted from unscaled (a) and scaled (b) data with the variance explained by these components (c,d) as well as the cumulative explained variance (e,f). PCA uses: (1) 100 principal components, (2) a window size of $150 \times 150$ samples and (3) 1000000 windows. 


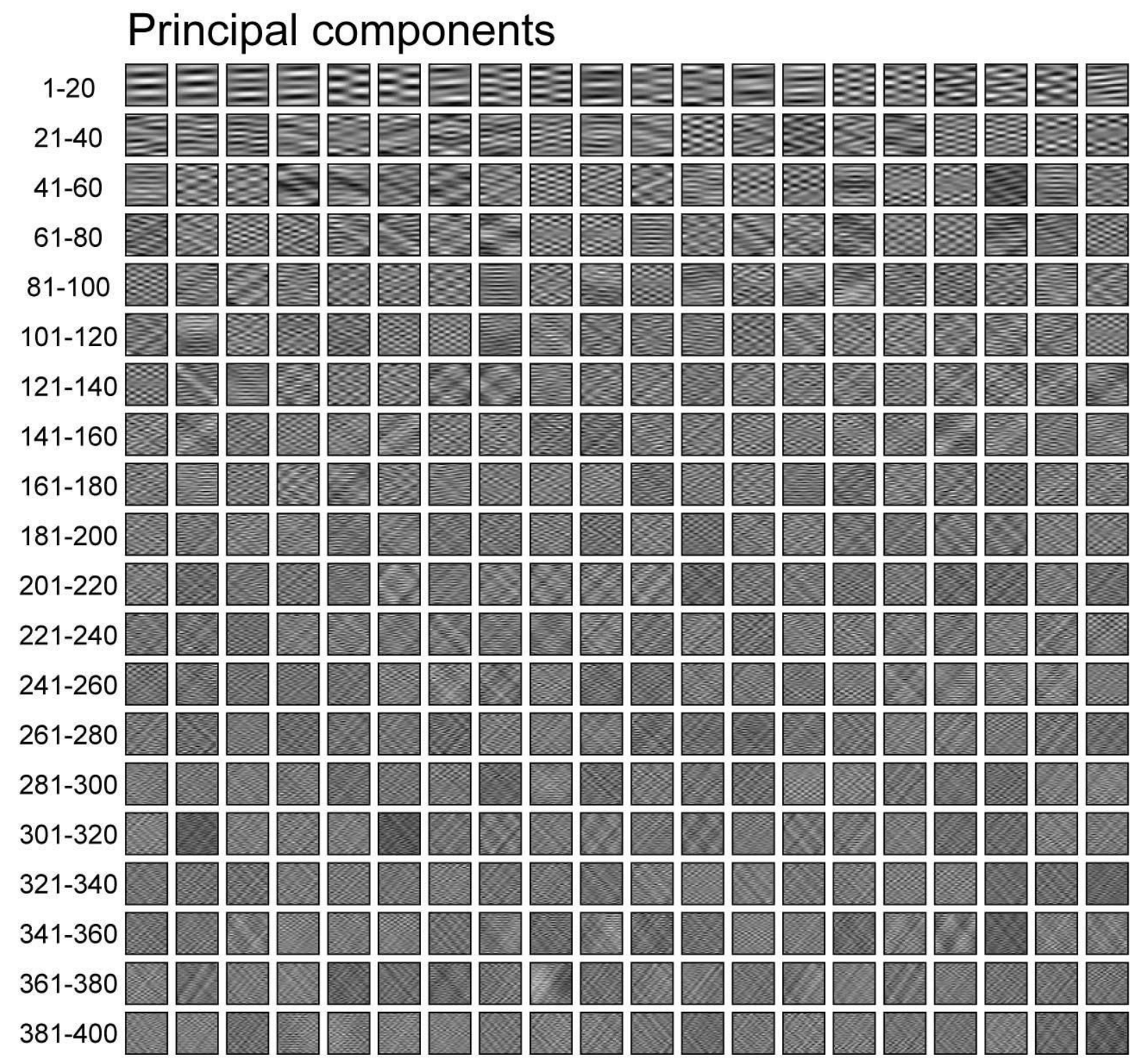

Figure 4: 400 principal components extracted from seismic section (Figure 2). PCA utilizes: (1) standardized data, (2) a window size of 150×150 samples and (3) 1000000 windows. Corresponding variance and cumulative variance explained by principal components are shown of Figure 5. Same color bar as Figure 2. 

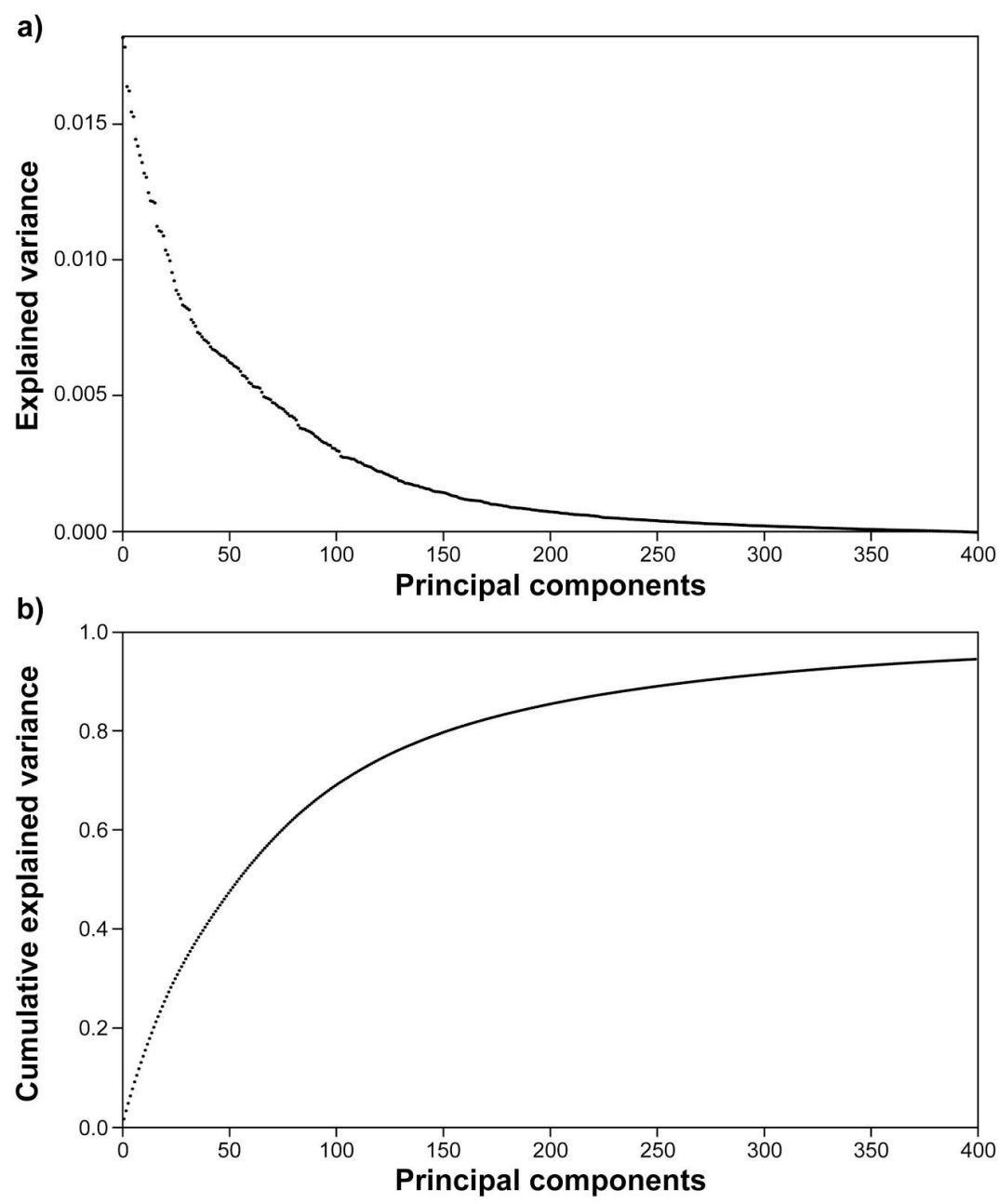

Figure 5: a) Variance and b) cumulative variance explained by principal components shown on Figure 4. PCA uses: (1) standardized data, (2) a window size of 150×150 samples and (3) 1000000 windows. 

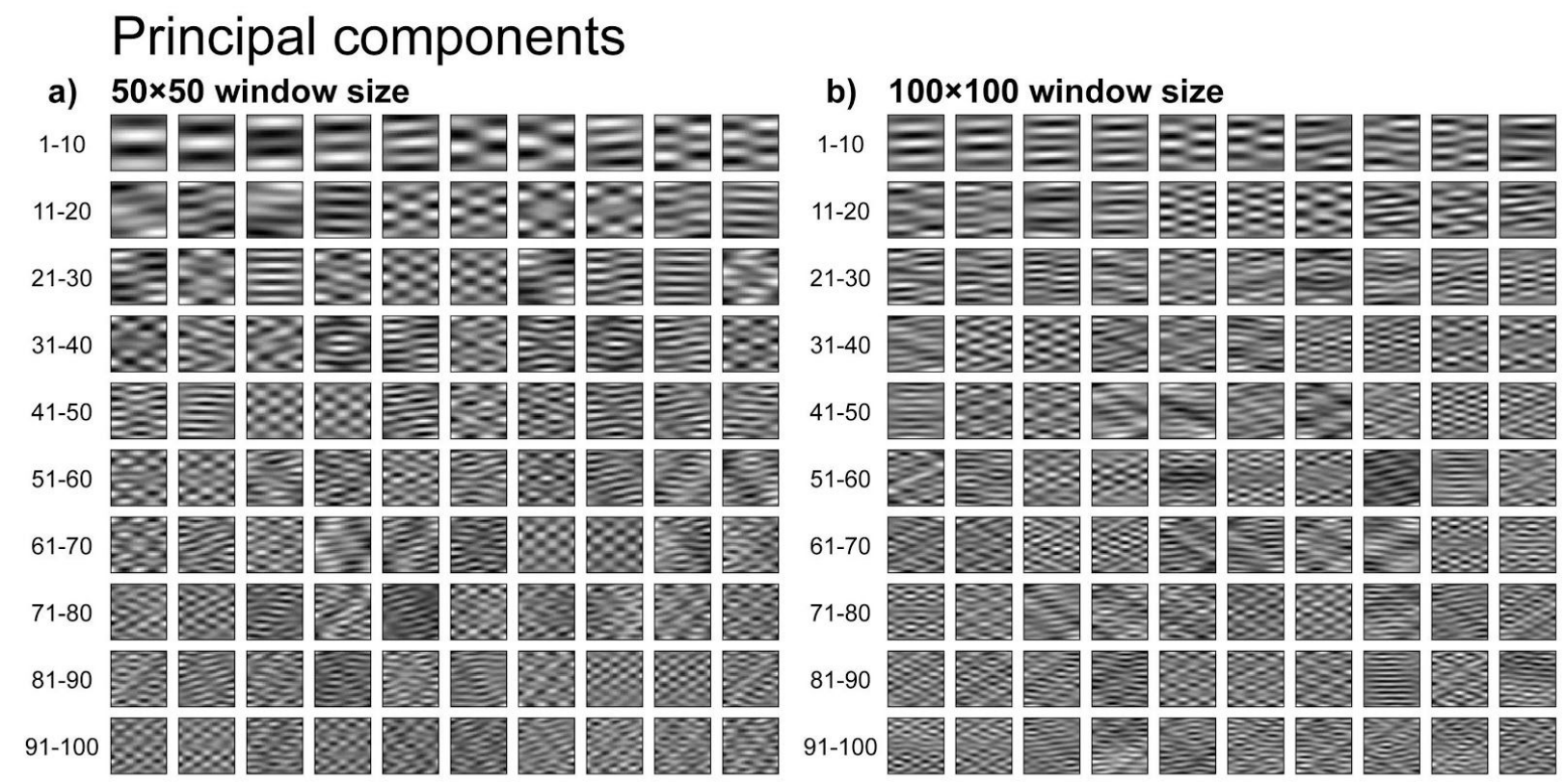

\section{c) $150 \times 150$ window size}

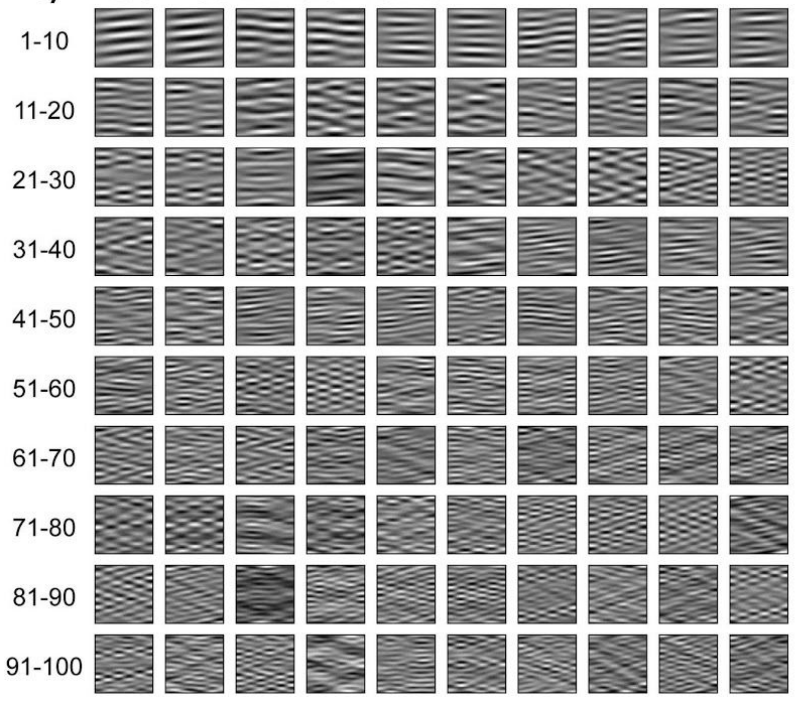

\section{d) $200 \times 200$ window size}

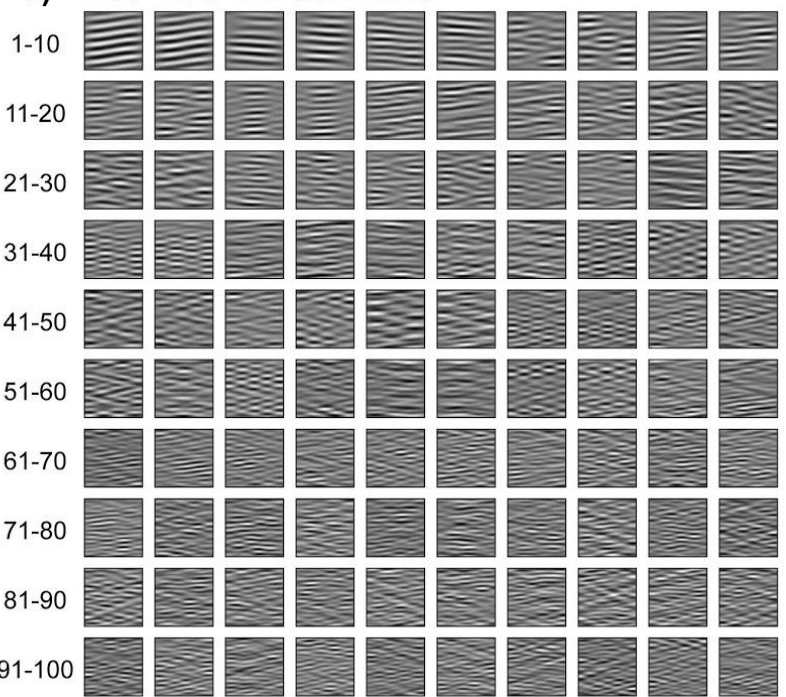

Figure 6: Principal components extracted with window sizes of a) $50 \times 50$, b) $100 \times 100$, c) $150 \times 150$ and d) $200 \times 200$ samples. PCA uses: (1) standardized data, (2) 100 principal components and (3) 1000000 windows. Corresponding cumulative variance explained by principal components is shown of Figure 7. Same color bar as Figure 1. 


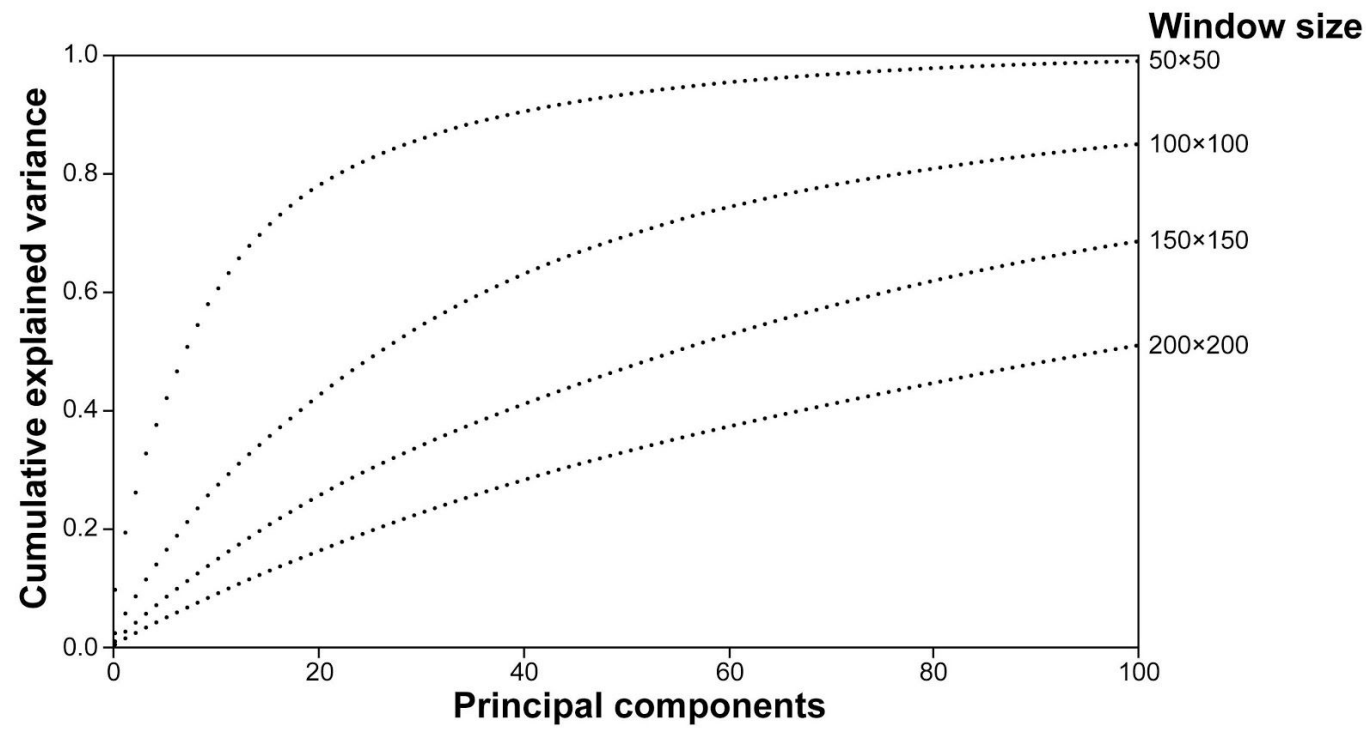

Figure 7: Cumulative variance explained by principal components shown on Figure 6. PCA uses: (1) standardized data, (2) 100 principal components and (3) 1000000 windows. 


\section{Principal components}

\section{a) 1000 windows}

1-10 $11-20 \cong \mathrm{E} \equiv$

$21-30$ E्:

$31-40$ 을

41.50 을

$51-60$ 굴 울

$61-70$

$71-80$

$81-90$

$91-100$

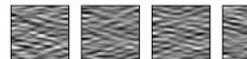

c) 100000 windows

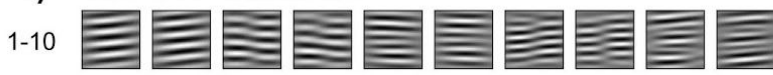

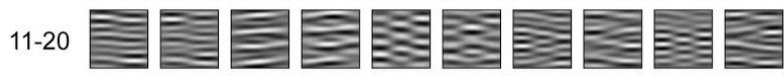

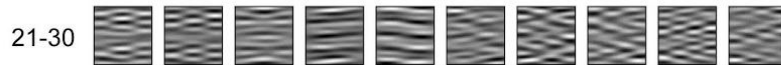

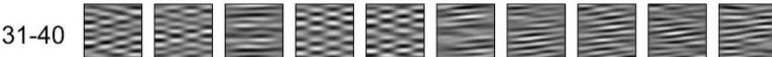

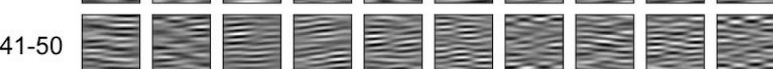

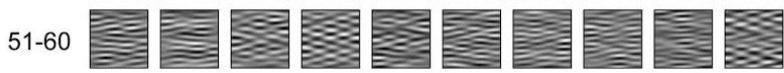

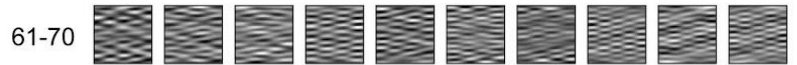

$71-80$ 을

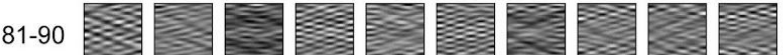

$91-100$ Pa b) 10000 windows

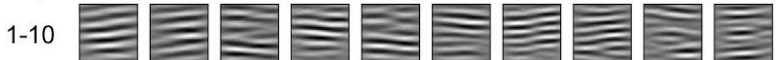

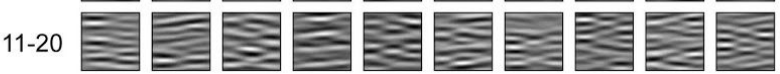

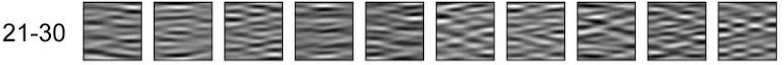

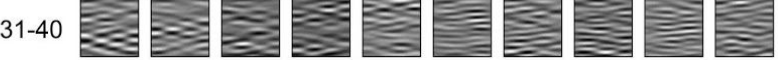

$41-50$ 坚

$51-60$ 晋

$61-70$ 管

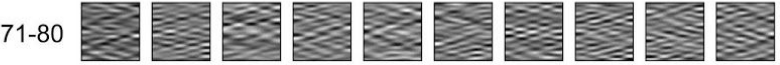

$81-90$ 10.

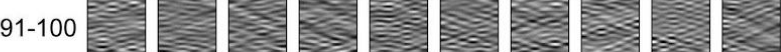

\section{d) 1000000 windows}

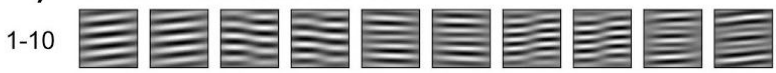

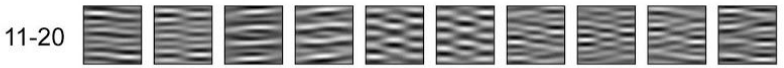

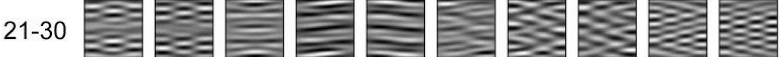

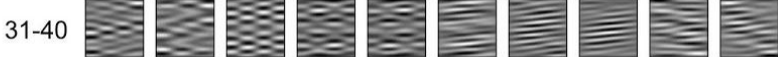

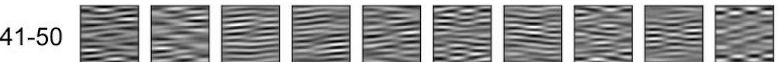

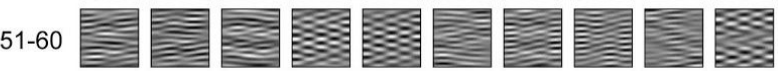

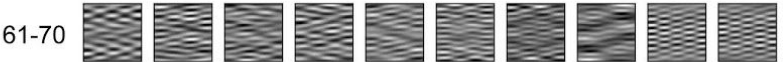

$71-80$ 698

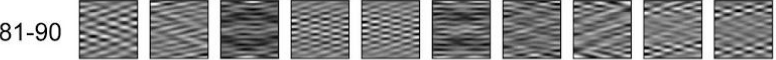

$91-100$ 술

Figure 8: Principal components extracted using $1000,10000,100000$ and 1000000 windows. PCA uses: (1) standardized data, (2) 100 principal components and (3) a window size of 100×100 samples. Cumulative explained variance is shown of Figure 6. Same color bar as Figure 1. 


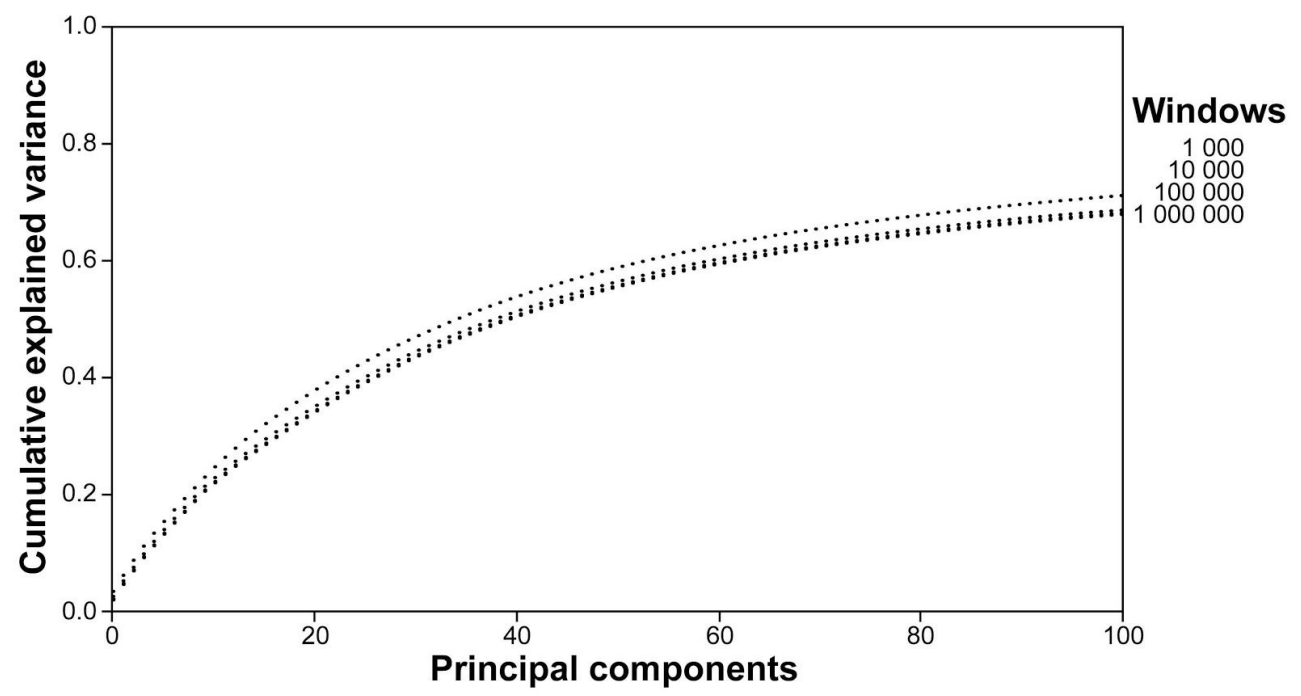

Figure 9: Cumulative explained variance calculated from $1000,10000,100000$ and 1000000 windows. PCA uses: (1) standardized data, (2) 100 principal components and (3) a window size of 150×150 samples. Corresponding principal components are shown of Figure 8.

After this sensitivity analysis, we apply PCA to the 2-D seismic section using: (1) standardized data, (2) 100 principal components, (3) a window size of 150×150 samples and (4) 1000000 windows. In addition to extracting the principal components, we can visualize the distribution of the components in the seismic section (Figure 10). For this purpose, we first project the window around each point of the seismic section onto each of the principal components. This gives us an idea of the importance of each component at each point. Next, we calculate the most 'important' principal component at each point, as the one with the highest absolute projection (Figure 10). This workflow provides us with a simple seismic facies map showing how much each principal component contributes to the seismic signal at each point of the data. 

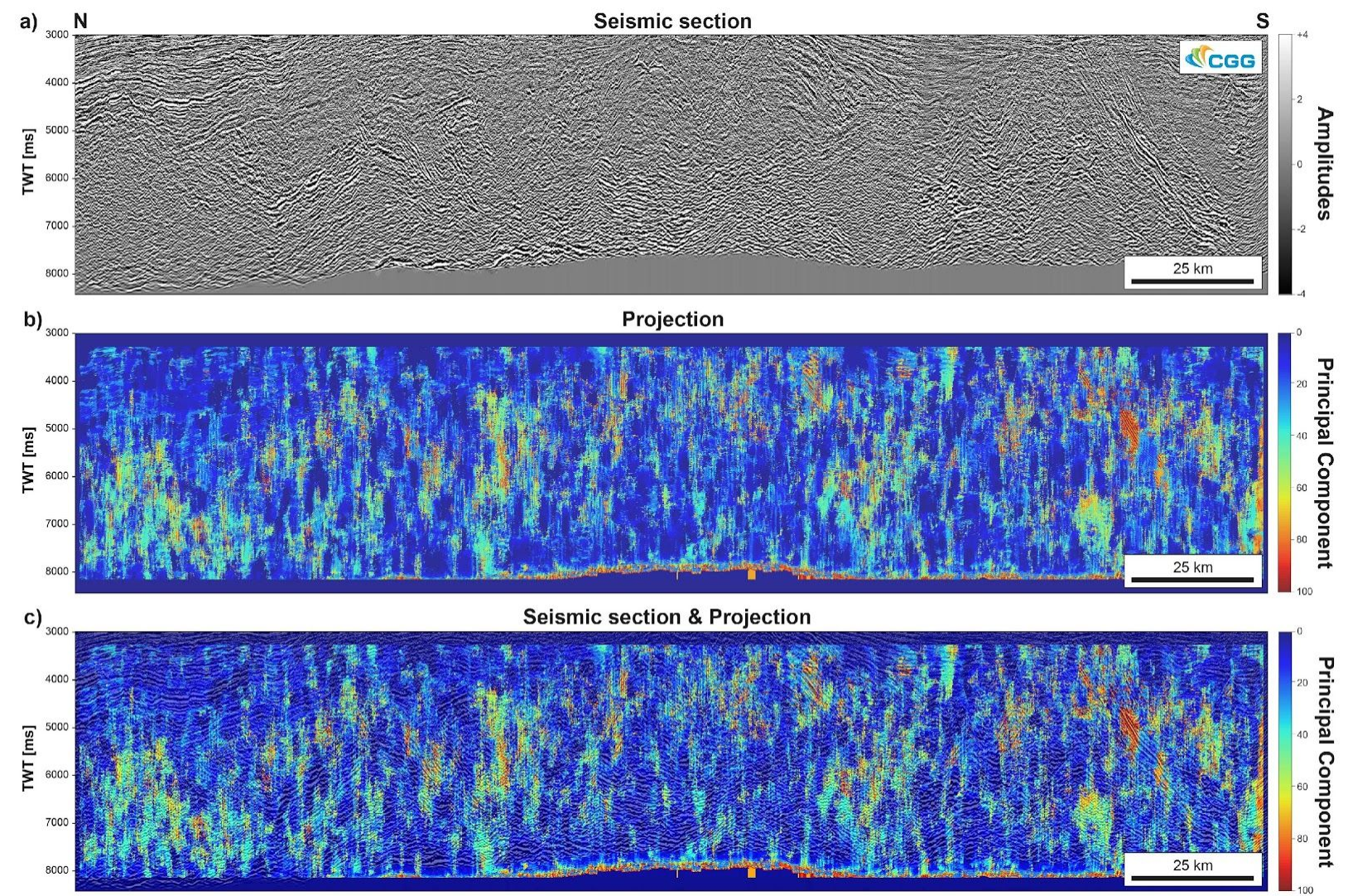

Figure 10: Comparison of original seismic section (top) and its dominant principal component (bottom). PCA uses: (1) standardized data, (2) 100 principal components, (3) a window size of 150×150 samples and (4) 1000000 windows. Seismic data courtesy of CGG.

\section{RESULTS}

Our results consist of principal components and plots showing the variance explained by each principal component (e.g. Figure 3). The principal components are numbered (1-100) and sorted with the first components in the upper left corner and the last one in the lower right corner of the plotted matrix. The variance explained by a principal component is the ratio between the variance of that principal component and the total variance in the data (i.e. all windows). As such, the explained variance describes how much of the total variance is explained by each principal component. The cumulative explained variance is the successive sum of the explained variance 
and thus describes how much of the total variance is explained by all principal components less and equal to this number (Figure 3e,f).

The sensitivity analysis produces results for different: (1) scaling, (2) numbers of principal components, (3) window sizes and (4) numbers of windows. First, standardized $(\mu=0, \sigma=1)$ or unstandardized data $(\mu=12, \sigma=18429696)$ produces the same results (Figure 3). This similarity is probably a result of the original seismic amplitudes already being close to zero mean $(\mu=12)$ relative to the large variance ( $\sigma=18429696$ ). Second, the higher the number of principal components, the higher the cumulative explained variance (Figures 4, 5). Early principal components explain most of the variance in the data (Figure 5). Early components also show typical seismic facies consisting of: (1) horizontal, (2) dipping, (3) displaced and (4) crisscrossing reflections (Figure 4). Third, smaller window sizes produce simpler patterns (Figure 6). In general, small windows contain less variance than large ones. Since PCA is able to capture more of the reduced variance from small windows, the cumulative explained variance is higher for small windows (Figure 7). Extracted seismic facies are thus simpler for smaller windows and more complex for larger ones (Figure 6). Intermediate window sizes of $150 \times 150$ samples allows us to extract typical seismic facies while capturing most of the variance $(>0.6)$ in the data (Figures $6,7)$. Fourth, the higher the number of windows, the clearer are the extracted seismic facies (Figure 8). Moreover, the cumulative explained variance converges with the number of windows (Figure 9).

Projecting the seismic reflection data on to the principal components shows that most of the variance in the data is explained by the early components (1-20), while less common features, such as inclined reflections, appear as higher components (60-100) (Figure 10). 


\section{DISCUSSION}

At this point, we examine the three basic assumptions underlying seismic facies analyses i.e. that seismic facies are: (1) well defined, (2) distinct from one another and (3) prevalent in the data. First, numerous studies define typical seismic facies showing: (1) horizontal, (2) dipping, (3) displaced and (4) crisscrossing reflections (e.g. Payton, 1977; Sheriff, 1980; Bally, 1987; Vail, 1987; Van Wagoner et al., 1987). In contrast, we are able to demonstrate that these facies, in fact, arise as the principal components of a large number of windows extracted from the data (e.g. Figure 4). As such, PCA offers a simple and fast way of extracting the main seismic facies from seismic reflection data.

Second, the extracted seismic facies are distinct from one another in the sense that the principal components are orthogonal. The principal components are, by definition, orthogonal (Shlens, 2014), i.e. the scalar product of any two components is zero. If we think of the scalar product as a measure of similarity, we see that any two principal components are dissimilar (Figures 3, 4, 6, 8). In this sense, all principal components are distinct from one another. We can also think about orthogonality in terms of linear combinations. Because the principal components are orthogonal to one another, we cannot express any one component (i.e. facies) as a linear combination of the others.

Third, PCA allows us to quantify how common the extracted seismic facies are in a given dataset. With PCA, we can calculate the variance explained by each principal component (e.g. Figure 5a). The cumulative variance explained by the principal components gives us an idea of the total variance in the data captured by PCA (e.g. Figure 5b). The steep initial increase in cumulative explained variance highlights that early components explain most variance while later ones explain less. For later components, the explained variance diminishes and the cumulative 
variance converges (Figures 3e,f; 5b; 7; 9). We can thus quantify how much of the variance in the data is explained by the principal components.

\section{APPLICATION}

After identifying the main seismic facies in the data, we would like to map them across the seismic section. This is typically done with machine learning, where PCA is used for feature extraction or dimensionality reduction. Since a full machine learning based facies classification goes beyond the scope of this paper, we simply show a way of visualizing where different principal components are dominant in the data. For this purpose, we first project the window around each point of the seismic section onto each of the principal components and then determine the principal components with the highest absolution projection (Figure 10). This calculation produces a simple seismic facies map showing how much each principal component contributes to the seismic signal at each point of the data.

\section{CONCLUSIONS}

This study demonstrates how to extract the main seismic facies from seismic reflection data using PCA. These seismic facies including: (1) horizontal, (2) dipping, (3) displaced and (4) crisscrossing reflections appear as the principal components of a large number (1000 000) of windows extracted from 2-D seismic reflection data. These seismic facies are distinct from one another (an important condition for seismic facies analyses) in the sense that the principal components are orthogonal. Analyzing the variance explained by each principal components (i.e. facies) reveals that it is possible to explain most of the variance in the data (>0.6) by a small number of seismic facies (100); a critical assumption for seismic facies analyses. Finally, we show a simple way to visualize these facies in a seismic section. 


\section{ACKNOWLEDGMENTS}

First, we would like to thank The Norwegian Academy of Science and Letters (VISTA) and The University of Bergen for supporting this project. We are very grateful to CGG for supplying seismic data and allowing us to publish this work. In particular, the support of Stein Åsheim and Marit Stokke Bauck is greatly appreciated. We thank the developers of python and scikit-learn, which was used to implement this workflow and we thank Leo Zijerveld for IT support.

\section{APPENDIX}

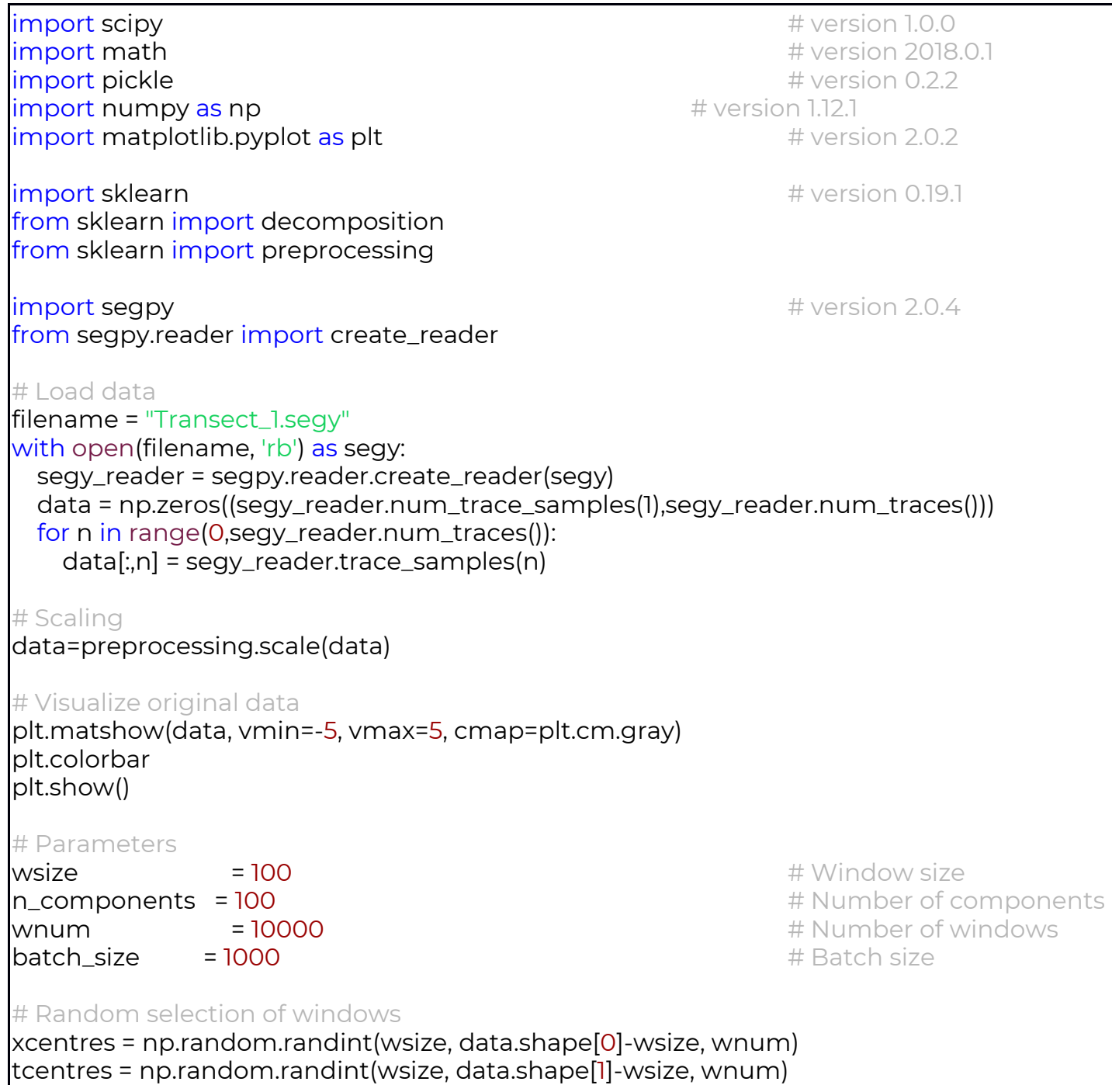




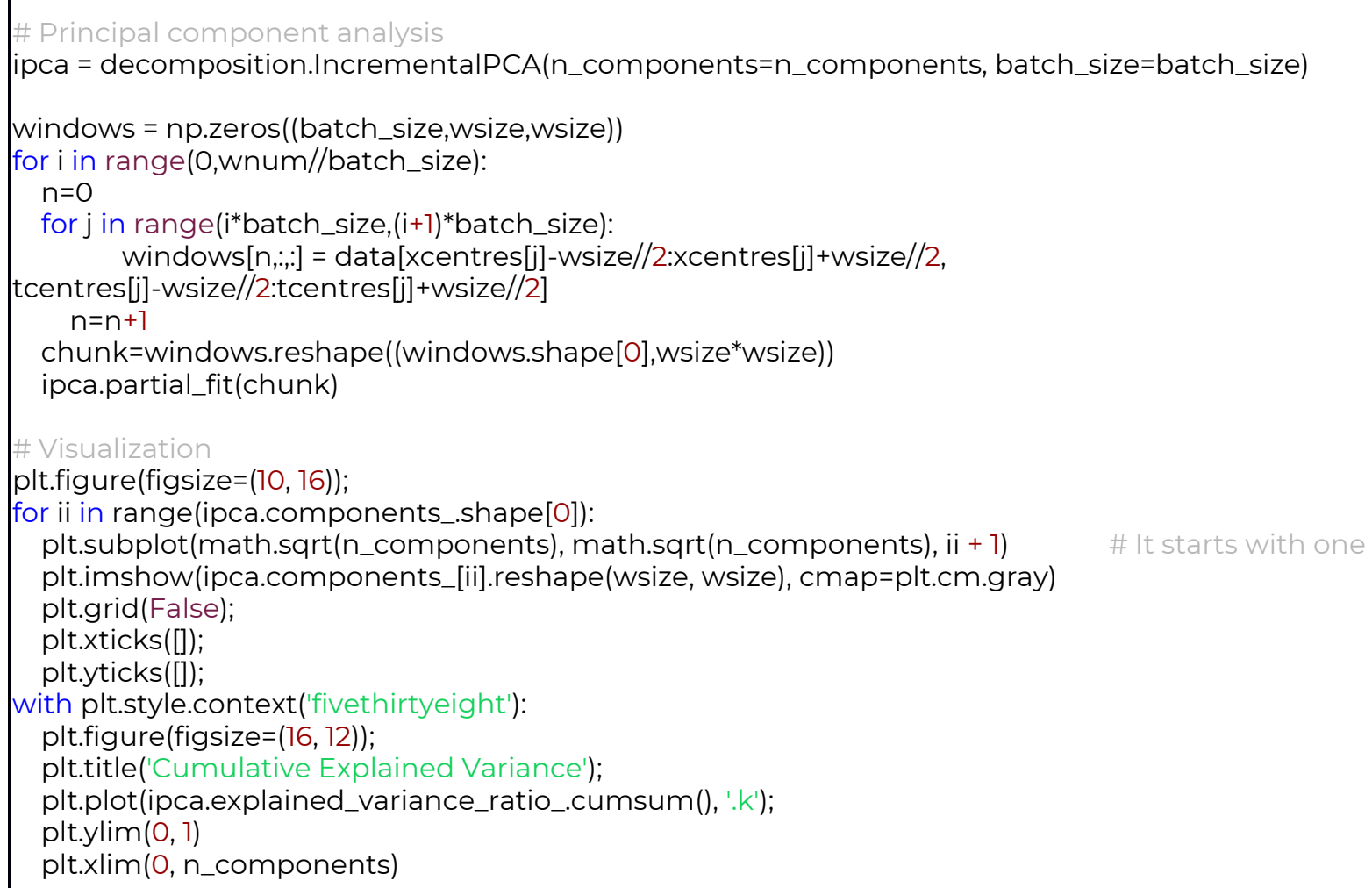

\section{REFERENCES}

Bally, A. W. 1987, Atlas of seismic stratigraphy.

Baudon, C., and J. A. Cartwright. 2008, 3D seismic characterisation of an array of blind normal faults in the Levant Basin, Eastern Mediterranean. Journal of Structural Geology, 30, no. 6,746-760. doi: DOI 10.1016/j.jsg.2007.12.008.

Bond, C. E. 2015, Uncertainty in structural interpretation: Lessons to be learnt. Journal of Structural Geology, 74,185-200. doi: 10.1016/j.jsg.2015.03.003.

Bond, C. E., R. Lunn, Z. Shipton, and A. Lunn. 2012, What makes an expert effective at interpreting seismic images? : Geology, 40, no. 1,75-78.

Bull, S., J. Cartwright, and M. Huuse. 2009, A review of kinematic indicators from mass-transport complexes using 3D seismic data. Marine and Petroleum Geology, 26, no. 7,1132-1151. doi: 10.1016/j.marpetgeo.2008.09.011. 
Cartwright, J., and M. Huuse. 2005, 3D seismic technology: the geological 'Hubble'. Basin Research, 17, no. 1,1-20.

Chopra, S., and K. J. Marfurt. 2007, Seismic attributes for prospect identification and reservoir characterization: Society of Exploration Geophysicists and European Association of Geoscientists and Engineers.

Chopra, S., and K. J. Marfurt. 2008, Emerging and future trends in seismic attributes. The Leading Edge, 27, no. 3,298-318.

Coléou, T., M. Poupon, and K. Azbel. 2003, Unsupervised seismic facies classification: A review and comparison of techniques and implementation. The Leading Edge, 22, no. 10,942-953.

Fazlikhani, H., H. Fossen, R. Gawthorpe, J. I. Faleide, and R. E. Bell. 2017, Basement structure and its influence on the structural configuration of the northern North Sea rift. Tectonics,n/a-n/a. doi: 10.1002/2017TC004514.

Firth, J., I. Horstad, and M. Schakel. 2014, Experiencing the full bandwidth of energy from exploration to production with the art of BroadSeis. First Break, 32, no. 6,89-97.

Hansen, D. M., J. A. Cartwright, and D. Thomas. 2004, 3D seismic analysis of the geometry of igneous sills and sill junction relationships. Geological Society, London, Memoirs, 29, no. 1,199-208.

Jolliffe, I. T. 1986, Principal component analysis and factor analysis, Principal component analysis: Springer. 115-128.

Macrae, E. J., C. E. Bond, Z. K. Shipton, and R. J. Lunn. 2016, Increasing the quality of seismic interpretation. Interpretation, 4, no. 3,T395-T402.

Marfurt, K. J., R. L. Kirlin, S. L. Farmer, and M. S. Bahorich. 1998, 3-D seismic attributes using a semblance-based coherency algorithm. Geophysics, 63, no. 4,1150-1165. doi: Doi 10.1190/1.1444415.

Morley, C. K. 2002, Evolution of large normal faults: Evidence from seismic reflection data. AAPG bulletin, 86, no. 6,961-978.

Payton, C. E. 1977, Seismic stratigraphy: applications to hydrocarbon exploration. Vol. 26: American Association of Petroleum Geologists Tulsa, OK.

Pedregosa, F., G. Varoquaux, A. Gramfort, V. Michel, B. Thirion, O. Grisel, M. Blondel, P. Prettenhofer, R. Weiss, V. Dubourg, J. Vanderplas, A. Passos, D. Cournapeau, M. 
Brucher, M. Perrot, and E. Duchesnay. 2011, Scikit-learn: Machine Learning in Python. Journal of Machine Learning Research, 12, no. Oct,2825-2830.

Planke, S., T. Rasmussen, S. Rey, and R. Myklebust. 2005, Seismic characteristics and distribution of volcanic intrusions and hydrothermal vent complexes in the Vøring and Møre basins. Paper read at Geological Society, London, Petroleum Geology Conference series.

Posamentier, H. W. 2004, Seismic geomorphology: imaging elements of depositional systems from shelf to deep basin using 3D seismic data: implications for exploration and development. Geological Society, London, Memoirs, 29, no. $1,11-24$.

Posamentier, H. W., and V. Kolla. 2003, Seismic geomorphology and stratigraphy of depositional elements in deep-water settings. Journal of Sedimentary Research, 73, no. 3,367-388. doi: Doi 10.1306/111302730367.

Roksandić, M. 1978, Seismic facies analysis concepts. Geophysical Prospecting, 26, no. 2,383-398.

Sheriff, R. 1980, Seismic Stratigraphy, published by International Human Resources Development Corporation. Boston, Massachusetts,85-116.

Sheriff, R. E. 2002, Encyclopedic Dictionary of Applied Geophysics, Encyclopedic Dictionary of Applied Geophysics.

Shlens, J. 2014, A tutorial on principal component analysis. arXiv preprint arXiv:1404.1100.

Turk, M. A., and A. P. Pentland. 1991, Face recognition using eigenfaces. Paper read at Computer Vision and Pattern Recognition, 1991. Proceedings CVPR'91., IEEE Computer Society Conference on.

Vail, P. R. 1987, Seismic stratigraphy interpretation using sequence stratigraphy: Part 1: Seismic stratigraphy interpretation procedure. AAPG Special Volumes, 27,1-10.

Van Wagoner, J., R. Mitchum Jr, H. Posamentier, and P. Vail. 1987, Seismic Stratigraphy Interpretation Using Sequence Stratigraphy: Part 2: Key Definitions of Sequence Stratigraphy.

Wold, S., K. Esbensen, and P. Geladi. 1987, Principal Component Analysis. Chemometrics and Intelligent Laboratory Systems, 2, no. 1-3,37-52. doi: Doi 10.1016/0169-7439(87)80084-9. 
